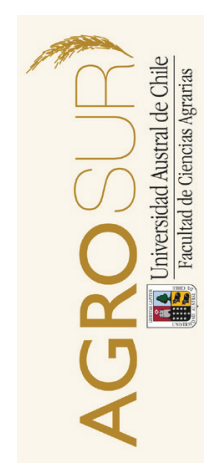

A RT ICLE INFO

Article history:

Received 13.08.2015

Accepted 02.04.2016

Keywords:

Grasses

Aluminum tolerance

Culture solution

Original Research Article,

Animal Science

${ }^{*}$ Corresponding author:

Sergio Radic

E-mail address:

sergio.radic@umag.cl;

dpinoche@uach.cl

\title{
Variación de la fitotoxicidad al aluminio en solución de ecotipos de bromo (Bromus valdivianus Phil.)
}

\author{
Variation of aluminum phytotoxicity in solution \\ of Bromus valdivianus Phil. ecotypes. \\ Radic, S. ${ }^{a *}$, Pinochet, D. ${ }^{b}$ Balocchi, 0. ${ }^{b}$ \\ ${ }^{a}$ Universidad de Magallanes, Facultad de Ciencias, Punta Arenas, Chile. \\ ${ }^{b}$ Universidad Austral de Chile, Facultad de Ciencias Agrarias, Valdivia, Chile.
}

\begin{abstract}
A B S T R A C T
The objective of this study was to evaluate the response of four ecotypes of Bromus valdivianus Phil. (41, 124, 12 and 58) to increasing levels of aluminum in solution. B. valdivianus ecotypes were previously selected using a solution screening methodology, then $\mathrm{Al}$ was applied into a solution in Leonard jars with quartz. Environmental conditions of temperature $\left(20^{\circ} \pm 3^{\circ} \mathrm{C}\right)$, relative humidity and photoperiod (16 h light; $8 \mathrm{~h}$ darkness) were controlled in the growth chamber. There were six treatments with four replications arranged in a completely random design. The nutrient solution used was renewed daily to maintain a constant $\mathrm{pH}$ of 4.2. The aluminum concentrations in solution were: $1.9 ; 5.7 ; 17.0 ; 51.0$ and $152.9 \mathrm{mg} \mathrm{Al} \mathrm{L}^{-1}$ (applied as aluminum sulfate) and a control, with no application of aluminum. The above ground and root dry matter produced was evaluated separately. To detect differences between treatments ( $p$-value $\leq 0.05$ ) analysis of variance was applied and Duncan test was used for differences of means.

The results showed that Bromus evaluated might present a mechanism of tolerance based on Al-root accumulation, suggested by the higher amount of Aluminum accumulated in their roots rather than the shoot. All the ecotypes evaluated showed a significant loss of dry matter production in the range of 5.7 and $17.0 \mathrm{mg} \mathrm{Al}^{-1}$. The increase in the aluminum concentration in the roots would depend on the tolerance level of the ecotype evaluated, suggesting that before dry matter yield loss there is an accumulation of aluminium in the root in tolerant ecotypes.
\end{abstract}

\section{RESUMEN}

El objetivo de este estudio fue evaluar el comportamiento de cuatro ecotipos (41, 124, 12 y 58) de Bromus valdivianus Phil. ante niveles crecientes de aluminio en solución aplicado a jarras tipo Leonard con cuarzo, estos ecotipos fueron seleccionados previamente a través de una metodología de selección en solución. Condiciones ambientales de temperatura $\left(20^{\circ} \pm 3^{\circ} \mathrm{C}\right)$, humedad relativa y fotoperíodo ( $16 \mathrm{~h}$ luz; $8 \mathrm{~h}$ oscuridad) fueron controladas en una cámara de crecimiento. Se utilizaron seis tratamientos con cuatro repeticiones, en un diseño completamente al azar. La solución nutritiva utilizada fue renovada diariamente, a pH constante de 4,2. Las concentraciones de aluminio en solución fueron: 1,9; 5,7; 17,0; 51,0 y 152,9 mg Al L-1 (como sulfato de aluminio) además de un control, sin aluminio. Se evaluó separadamente la parte aérea y radical para la materia seca producida. Los resultados fueron estadísticamente analizados a través de un análisis de varianza ( $\mathrm{p}$-valor $\leq 0,05)$, aplicando el test de Duncan para evaluar diferencias entre medias.

Los resultados evidenciaron que los cuatro ecotipos de la especie Bromus presentarían un mecanismo de tolerancia clasificado como Al-acumulador radical, sugerido por la mayor acumulación del aluminio en la parte radical que en la parte aérea. En el rango de 5,7 y 17,0 $\mathrm{mg} \mathrm{Al}^{-1}$, se produciría una pérdida significativa en producción de materia seca para todos los ecotipos evaluados. El aumento en concentración de aluminio radical dependería del grado de tolerancia del ecotipo, sugiriendo que la acumulación de aluminio radical, en ecotipos tolerantes, se produce antes de la pérdida en productividad de materia seca.

Palabras clave: gramíneas, tolerancia al aluminio y solución nutritiva.

\section{INTRODUCIÓN}

La acidez de los suelos, es el mayor factor limitante de crecimiento para las plantas (Foy, 1984). Alrededor del 25 al $30 \%$ de los suelos del mundo son ácidos y representan las regiones más importantes en producción de alimentos (Havlin et al., 2005). Explicaciones del pobre crecimiento de las plantas en suelos ácidos, que se relacionan con la toxicidad por aluminio (Al), han sido incluidas por Kinraide (1993); Marschner (1995); Barceló (1996); George et al. (2012) y Yang et al. (2013). Según Marschner (1991), la inhibición del crecimiento 
de las plantas resulta de una variedad de factores químicos específicos e interacciones entre estos factores. En los suelos minerales ácidos los mayores problemas para el desarrollo de las plantas son el incremento en la concentración de $\mathrm{H}^{+}$(toxicidad por $\mathrm{H}^{+}$); el incremento en la concentración de aluminio (toxicidad por aluminio); el incremento en la concentración de manganeso (toxicidad por manganeso); la disminución en la concentración de macronutrientes cationes (deficiencias de magnesio, calcio y potasio); la disminución del fósforo y molibdeno (deficiencia de fósforo y molibdeno); la inhibición en el crecimiento de raíces y la absorción de agua (deficiencia de nutrientes, estrés por sequía e incremento de la lixiviación de nutrientes). Este último punto, explicado en detalle por Yang et al. (2013).

En la mayoría de las especies, la concentración del $\mathrm{Al}$ en las raíces y su inhabilitación a ser transportado hacia la parte aérea, pareciera ser un mecanismo común de adaptación a la fitotoxicidad del Al (Bennet y Breen, 1991). Dentro de los mecanismos de las plantas para evitar el daño por aluminio se pueden mencionar la tolerancia y la exclusión, de estos dos mecanismos, el primero confiere la habilidad a las plantas para tolerar el $\mathrm{Al}$ en su interior, específicamente en el simplasma de las plantas y el segundo facilita la exclusión del Al desde el ápice radicular o su ingreso a la raíz (Kochian, 1995; Kochian et al., 2005). Cada una de estas estrategias de adaptación de las plantas a los suelos ácidos tiene características específicas. Las plantas que toleran el aluminio, poseen una baja demanda interna de nutrientes, una compartimentalización y recirculación de nutrientes minerales y una alta tolerancia de los tejidos a los elementos tóxicos ( $\mathrm{Al}$ y $\mathrm{Mn}$ ). Mientras que las plantas que excluyen el aluminio, incrementan la adquisición de nutrientes minerales, inducen cambios en la rizósfera y excluyen $\mathrm{Al}$ desde sitios sensitivos y de captación (el citoplasma, la interfase membrana plasmática-apoplasma y la cofia). Dentro de las dos principales estrategias de las plantas para la adaptación a condiciones adversas de suelo (tolerancia y exclusión), la estrategia de exclusión es la más común para la adaptación de suelos minerales ácidos (Marschner, 1991; George et al., 2012; Ma et al., 2014).

Bromus valdivianus Phil. (Bromo) es una especie perenne nativa del sur de Chile, que crece en praderas naturales (Matthei, 1986) y se encuentra en el dominio húmedo (López et al., 1997). En estudios previos (López et al., 1997) se ha determinado que el bromo es una especie que domina en sitios con bajo $\mathrm{Al}$ intercambiable. Sin embargo, al ser nativa del sur de Chile puede presentar una gran variación en la sensibilidad $\mathrm{Al}$ en los diferentes ecotipos presentes en la región (Forde y Edgar, 1995; Stewart, 1996). Es por lo anterior, que el objetivo de este estudio fue evaluar el comportamiento de cuatro ecotipos de Bromus valdivianus Phil. ante niveles crecientes de aluminio en solución.

\section{MATERIALES Y MÉTODOS}

Bajo condiciones controladas se estudió el efecto de la aplicación de soluciones nutritivas con y sin adición de aluminio a ecotipos seleccionados de bromo. Los ecotipos corresponden a una colecta de plantas de bromo de la Región de Los Ríos caracterizada por Blanco et al. (2010). En este experimento, el período de crecimiento de las plantas fue de 73 días, durante los cuales fueron evaluadas con adición de aluminio por 61 días. La temperatura de la cámara de crecimiento fue controlada con un sistema de aire acondicionado, regulado a una temperatura promedio de $20^{\circ} \mathrm{C} \pm 3^{\circ} \mathrm{C}$. La humedad relativa durante el ensayo se registró a través de un higrómetro y fue de $64 \%$. Se utilizó un fotoperíodo de 16 horas de luz y 8 horas de oscuridad. Con una intensidad promedio de luz 12.000 lux. La especie en este experimento correspondió a Bromus valdivianus Phil; y los ecotipos elegidos fueron determinados en base a un trabajo de selección previo. En este trabajo previo (Toneatti, 2006) se seleccionaron y clasificaron de acuerdo a su sensibilidad o tolerancia al aluminio 125 ecotipos de bromo, de los cuales se utilizaron en este ensayo el $n^{\circ} 41$ y 124 (clasificados como tolerantes al aluminio); el $\mathrm{n}^{\circ} 12$ (de tolerancia media); y el $\mathrm{n}^{\circ} 58$ (sensible). Se utilizaron Jarras Leonard confeccionadas a partir de botellas de $750 \mathrm{~cm}^{3}$ de capacidad, las cuales, fueron cortadas por la mitad. La parte superior de las jarras, donde fueron desarrolladas las plantas, presentaban un alto de $8,5 \mathrm{~cm}$ y un área de $40,7 \mathrm{~cm}^{2}$ lo que corresponde a un volumen de $346 \mathrm{~cm}^{3}$. Las jarras fueron lavadas inicialmente con una solución diluida de ácido clorhídrico (5\%). Posteriormente el material fue sometido a lavados sucesivos con agua destilada. Como sustrato se utilizó cuarzo, previamente lavado con ácido clorhídrico $(8,5 \%)$, seguido de sucesivos lavados con agua destilada. Posteriormente, el cuarzo se secó al sol en los mesones del laboratorio. Finalmente, se determinó el aluminio intercambiable en el sustrato utilizado en el ensayo, por medio del método con $\mathrm{KCl}$ 1 molar, descartando cualquier posible contaminación.

Las semillas fueron germinadas sobre papel filtro saturado con una solución al 0,2\% de $\mathrm{KNO}_{3}$ en un germinador tipo Jacobsen. La duración total en la etapa de germinación fue de 15 días. Se utilizó la composición de la solución nutritiva utilizada por Rengel y Robinson (1989). Las soluciones nutritivas fueron renovadas diariamente, utilizando un volumen aproximado de $125 \mathrm{~mL}$ por jarra. Una vez por semana se controló el $\mathrm{pH}$, para mantener un nivel constante de 4,2. Utilizando hidróxido de sodio $(\mathrm{NaOH})$ cuando el pH fuera ácido y ácido clorhídrico $(\mathrm{HCl})$ cuando el $\mathrm{pH}$ fuera básico. Las dosis que se utilizaron fueron de 1,9-5,7 -17 -51 $-152,9 \mathrm{mg} \mathrm{Al} \mathrm{L}^{-1}(70-210-630-1890-5670 \mu \mathrm{mol} \mathrm{Al}$ $\mathrm{L}^{-1}$ ) además de un control, sin aplicación de aluminio. El Al fue aplicado como sulfato de aluminio hidratado 
$\left(\mathrm{Al}_{2}\left(\mathrm{SO}_{4}\right)_{3} \times 18 \mathrm{H}_{2} \mathrm{O}\right)$. Se midieron las variables de producción de materia seca (mg jarra-1) y concentración del aluminio ( $\mathrm{mg} \mathrm{kg}^{-1}$ de MS) tanto en la parte aérea como radical. Las plantas fueron cosechadas completas desde las jarras y sometidas a baños con agua destilada en su parte aérea y radical. Posteriormente, el material vegetal fue secado a $60^{\circ} \mathrm{C}$ por $48 \mathrm{~h}$, determinándose en base a peso la materia seca (MS). Las muestras fueron calcinadas en una mufla a $550{ }^{\circ} \mathrm{C}$ por 6 horas. En las cenizas se determinó el aluminio utilizando espectrofotometría de absorción atómica.

El ensayo se manejó como un experimento completamente al azar, con cinco tratamientos más un control sin adición de aluminio, para cada uno de los cuatro ecotipos evaluados y cada tratamiento tuvo cuatro repeticiones. Para detectar si existieron diferencias significativas entre los tratamientos se realizó un análisis de varianza de Fisher, utilizándose el test de Duncan (p-valor $<0,05$ ) para la separación de medias, cuando existieron diferencias estadísticas. Para determinar el comportamiento de las gramíneas evaluadas a través de los distintos tratamientos con aluminio se realizó un análisis de regresión no lineal.

El análisis de regresión entre las variables de aluminio en solución (mg Al L ${ }^{-1}$ ) y materia seca (mg jarra $\left.{ }^{-1}\right)$ se analizó en función del siguiente modelo, para los parámetros de materia seca aérea y radical.

$$
\text { Modelo cuadrático inverso: } \mathrm{Y}=\mathrm{A}+\mathrm{B} / \mathrm{X}+\mathrm{C} / \mathrm{X}^{2}
$$

Se utilizó una sola ecuación para ajustar los datos de producción de materia seca radical y aérea, en relación al aluminio aplicado en solución. Debido a que ajustar la misma ecuación para todas las gramíneas, la posibilidad de compararlas es mucho mayor.

El análisis de regresión entre las variables de aluminio en solución ( $\mathrm{mg} \mathrm{Al} \mathrm{L}^{-1}$ ) y concentración de aluminio $\left(\mathrm{mg} \mathrm{kg}^{-1}\right)$ se analizó en función del modelo cuadrático (2) para los parámetros de concentración de Al radical, mientras que la concentración de $\mathrm{Al}$ aéreo se privilegió la ecuación cuadrática eligiéndose otra ecuación en aquellas gramíneas que se desviaron significativamente de este modelo. Para el parámetro de concentración aérea en los ecotipos 124 y 58 se utilizó un modelo cuadrático (2). Para el bromo 12 se utilizó el modelo lineal (3) y para el bromo 41 se utilizó el modelo doble potencial (4). El criterio de selección de las ecuaciones a utilizar fue considerando el coeficiente de determinación (Cuadro 4), la suma de cuadrados del error y el sentido biológico de cada ecuación.

$$
\begin{array}{ll}
\text { Modelo cuadrático: } & \mathrm{Y}=\mathrm{A}+\mathrm{BX}+\mathrm{CX}^{2} \\
\text { Modelo lineal: } & \mathrm{Y}=\mathrm{A}+\mathrm{BX} \\
\text { Modelo doble potencial: } & \mathrm{Y}=\mathrm{AX}^{\mathrm{B}}+\mathrm{CX}^{\mathrm{D}}
\end{array}
$$

\section{RESULTADOS Y DISCUSIÓN.}

La producción de materia seca aérea y radical de los ecotipos evaluados de bromo disminuyó, como aumentaba el Al aplicado en solución, efectos similares encontraron Rengel y Robinson (1989) evaluando diferentes cultivares de ballica (Lolium multiflorum Lam.) en un experimento de aplicación de aluminio en soluciones nutritivas. Reforzando la idea anterior, diversos autores señalan la disminución de la materia seca aérea y radical por efecto del Al solución (Mossor-Pietraszewska, 2001; Menzies, 2003; Havlin et al., 2005; Vardar y Ünal, 2007; Brady y Weil, 2008; George et al., 2012). En la producción de materia seca de la parte aérea se muestra que al aumentar el aluminio en solución (Cuadro 1) disminuye la producción de MS en todos los ecotipos de bromo evaluados, presentándose esta disminución en el rango de 5,7 y $17 \mathrm{mg} \mathrm{Al} \mathrm{L}^{-1}$.

Resultados similares en gramíneas fueron encontrados por Wheeler et al. (1992b) en un experimento con $\mathrm{Al}$ en solución. No se encontraron diferencias estadísticas entre el primer $\left(0 \mathrm{mg} \mathrm{Al} \mathrm{L}^{-1}\right)$ y tercer tratamiento $\left(5,7 \mathrm{mg} \mathrm{Al} \mathrm{L}^{-1}\right)$ para todos los ecotipos de bromo. Esta falta de significancia estadística para los primeros tratamientos, pudo deberse a la tendencia de una mayor acumulación de MS aérea, sin ser estadísticamente significativa, con la dosis de $1,9 \mathrm{mg} \mathrm{Al} \mathrm{L}^{-1}$ para los ecotipos de bromo $12 \mathrm{y}$ 58. Solo a partir del cuarto tratamiento $(17,0 \mathrm{mg} \mathrm{Al}$ $\mathrm{L}^{-1}$ ), se presentan diferencias significativas para todos los ecotipos. Variando las diferencias en producción de MS para los diferentes ecotipos dependiendo del tratamiento, pero indicando una mayor producción de los ecotipos más tolerantes ( 41 y 124). También se puede observar que en el tratamiento testigo, los menos tolerantes producen menor MS, pero responden bien a la adición de $\mathrm{Al}$, al menos en el primer tramo. Pero esto no puede ser concluyente dado la falta de significancia en producción de MS aérea para esos tratamientos en todos los ecotipos evaluados, de todas formas, este comportamiento puede evaluarse en futuros ensayos.

Otra explicación para este comportamiento de aumento de producción de MS en los primeros tratamientos pudo ser, por un efecto benéfico del $\mathrm{Al}$ a bajas concentraciones, como se refiere Marschner (1995) y Broadly et al. (2012), los cuales, señalan que concentraciones de $\mathrm{Al}$ en solución entre $71,4 \mu \mathrm{M}(1,93 \mathrm{mg} \mathrm{Al}$ $\left.\mathrm{L}^{-1}\right)$ y $185 \mu \mathrm{M}\left(4,99 \mathrm{mg} \mathrm{Al} \mathrm{L}^{-1}\right)$ producen una estimulación del crecimiento dependiendo de la especie.

Se observa en el cuadro 1, que la producción de materia seca de la parte radical en todos los ecotipos de bromo evaluados, disminuye al aumentar el aluminio en solución, no existiendo diferencias estadísticas significativas entre el primer $\left(0 \mathrm{mg} \mathrm{Al} \mathrm{L}^{-1}\right)$ y tercer tratamiento $\left(5,7 \mathrm{mg} \mathrm{Al} \mathrm{L}^{-1}\right)$. Exceptuando el ecotipo 58, el cual presenta diferencias significativas a partir del tercer 
Cuadro 1. Producción promedio de materia seca aérea y radical (mg MS jarra-1) en los cuatro ecotipos de Bromus valdivianus $(41,124,12$ y 58$)$.

Table 1. Shoot and root mass production (mg DM jar-1) of the four ecotypes of Bromus valdivianus $(41,124,12$, and 58).

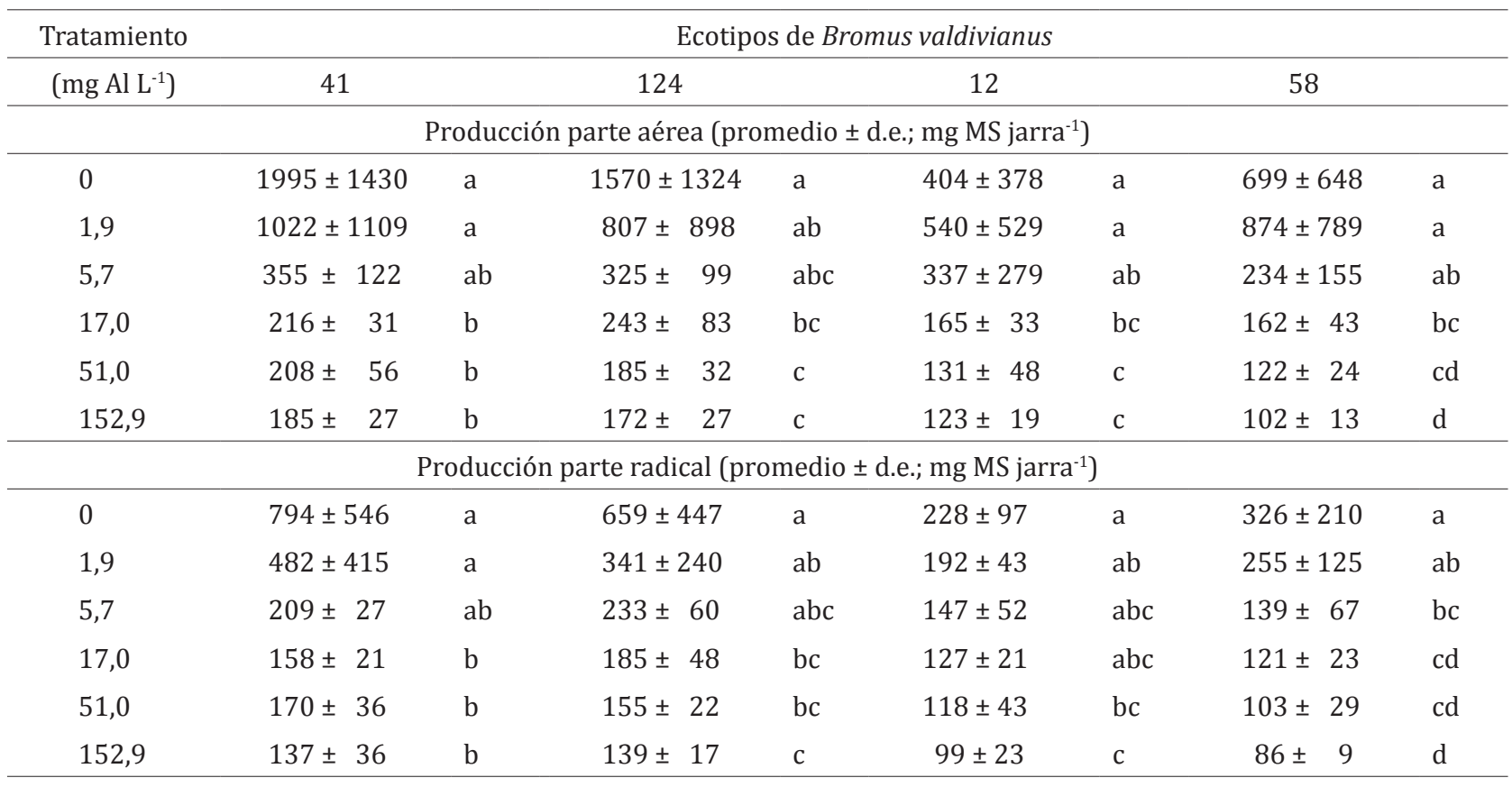

Diferentes letras dentro de cada columna indica diferencias significativas (p-valor $\leq 0,05$; Duncan); d.e.= Desviación estándar.

tratamiento. En el cuarto tratamiento $\left(17,0 \mathrm{mg} \mathrm{Al} \mathrm{L}^{-1}\right)$ se presentan diferencias significativas para los ecotipos 41 y 124 , para finalizar en el quinto tratamiento $(51,0$ mg Al L'-1) en el ecotipo 12. Los resultados de la producción de materia seca radical presentan comportamientos similares a las existentes en la producción de materia seca aérea, aunque la magnitud de la producción radical es menor. Resultados similares fueron encontrados por Rengel y Robinson (1989) y De Macedo et al. (2011). Vardar y Ünal (2007) y Brady y Weil (2008), señalan la disminución del tamaño radical como consecuencia del aluminio en solución. Hayes et al. (2011), señalan que en un experimento con alfalfa en solución nutritiva, la tolerancia se indicó por el aumento en el largo de las raíces, mientras las más afectadas tuvieron una disminución en el peso y largo.

En términos generales, los altos coeficientes de determinación obtenidos (Cuadro 2) para la misma ecuación (Ec. 1) sugieren que la variación en los parámetros evaluados es similar y más dependiente de la especie que de los tratamientos estudiados, además, sugiriendo que los mecanismos de respuesta en productividad son similares. Sin embargo, las variaciones en los parámetros de la ecuación ajustada permitirían diferenciar el grado de la respuesta en producción y la variación que podrían presentar los distintos ecotipos evaluados.

En lo matemático, el modelo (Ec. 1) muestra que Y decrece de manera no lineal a medida que $\mathrm{X}$ aumenta a partir de un máximo alcanzado. Desde este máximo existe un mayor efecto de la variable independiente sobre la dependiente en valores menores que en los valores mayores. Desde el punto de vista biológico, esta ecuación describe el efecto de la toxicidad producida por el incremento de Al en solución, sobre la producción de materia seca para todos los ecotipos evaluados.

Las concentraciones de aluminio de la parte aérea fueron inferiores en promedio a los de la parte radical para todos los ecotipos de bromo evaluados, este efecto es observable en cada tratamiento aplicado, resultados similares en Trifolium repens L. para un experimento con aluminio en solución, fueron encontrados por Lee y Pritchard (1984), demostrando con esto, que efectos similares de la toxicidad por aluminio se pueden dar en diferentes familias. Silva (2012), señala también la mayor concentración de $\mathrm{Al}$ en la parte radical que en la parte aérea en algunas especies Al-acumuladoras. En el cuadro 3, se muestra que al aumentar el Al en solución aumenta el Al contenido en la parte aérea. Sin embargo, las diferencias observadas fueron estadísticamente significativas para los ecotipos de bromo 41, 124 y 58, mientras que el ecotipo de bromo 12 no presentó diferencias significativas. La falta de significancia estadística podría deberse a que las concentraciones de aluminio en la parte aérea fueron relativamente bajas y con un alto coeficiente de variación, 
Cuadro 2. Coeficientes de regresión y determinación para producción de materia seca aérea y radical de ecotipos de B. valdivianus.

Table 2. Regression and determination coefficients for shoot and root mass production of four Bromus valdivianus ecotypes.

\begin{tabular}{|c|c|c|c|c|}
\hline \multirow[b]{2}{*}{ Ecotipos } & \multicolumn{4}{|c|}{ Parámetros de la ecuación ajustada } \\
\hline & A & $\mathrm{B}$ & $\mathrm{C}$ & $\mathrm{R}^{2}$ \\
\hline & \multicolumn{4}{|c|}{ Materia seca aérea } \\
\hline Bromo 41 & 0,2091 & 0,4599 & $-0,004364$ & 0,998 \\
\hline Bromo 124 & 0,2042 & 0,3356 & $-0,003308$ & 0,964 \\
\hline Bromo 12 & 0,1311 & 0,8166 & $-0,008157$ & 0,962 \\
\hline \multirow[t]{2}{*}{ Bromo 58} & 0,1378 & 0,2233 & $-0,002177$ & 0,958 \\
\hline & \multicolumn{4}{|c|}{ Materia seca radical } \\
\hline Bromo 41 & 0,1528 & 0,2110 & $-0,002028$ & 0,999 \\
\hline Bromo 124 & 0,1653 & 0,1356 & $-0,001338$ & 0,876 \\
\hline Bromo 12 & 0,1115 & 0,1592 & $-0,001585$ & 0,955 \\
\hline Bromo 58 & 0,1030 & 0,1185 & $-0,001162$ & 0,953 \\
\hline
\end{tabular}

Cuadro 3. Concentración promedio de aluminio en la parte aérea y radical ( $\mathrm{mg} \mathrm{Al} \mathrm{kg}{ }^{-1}$ ) en los cuatro ecotipos de Bromus valdivianus $(41,124,12$ y 58$)$.

Table 3. Shoot and root Aluminum concentrations ( $\left.\mathrm{mg} \mathrm{Al} \mathrm{kg}^{-1}\right)$ in the four ecotypes of Bromus valdivianus $(41,124,12$, and 58).

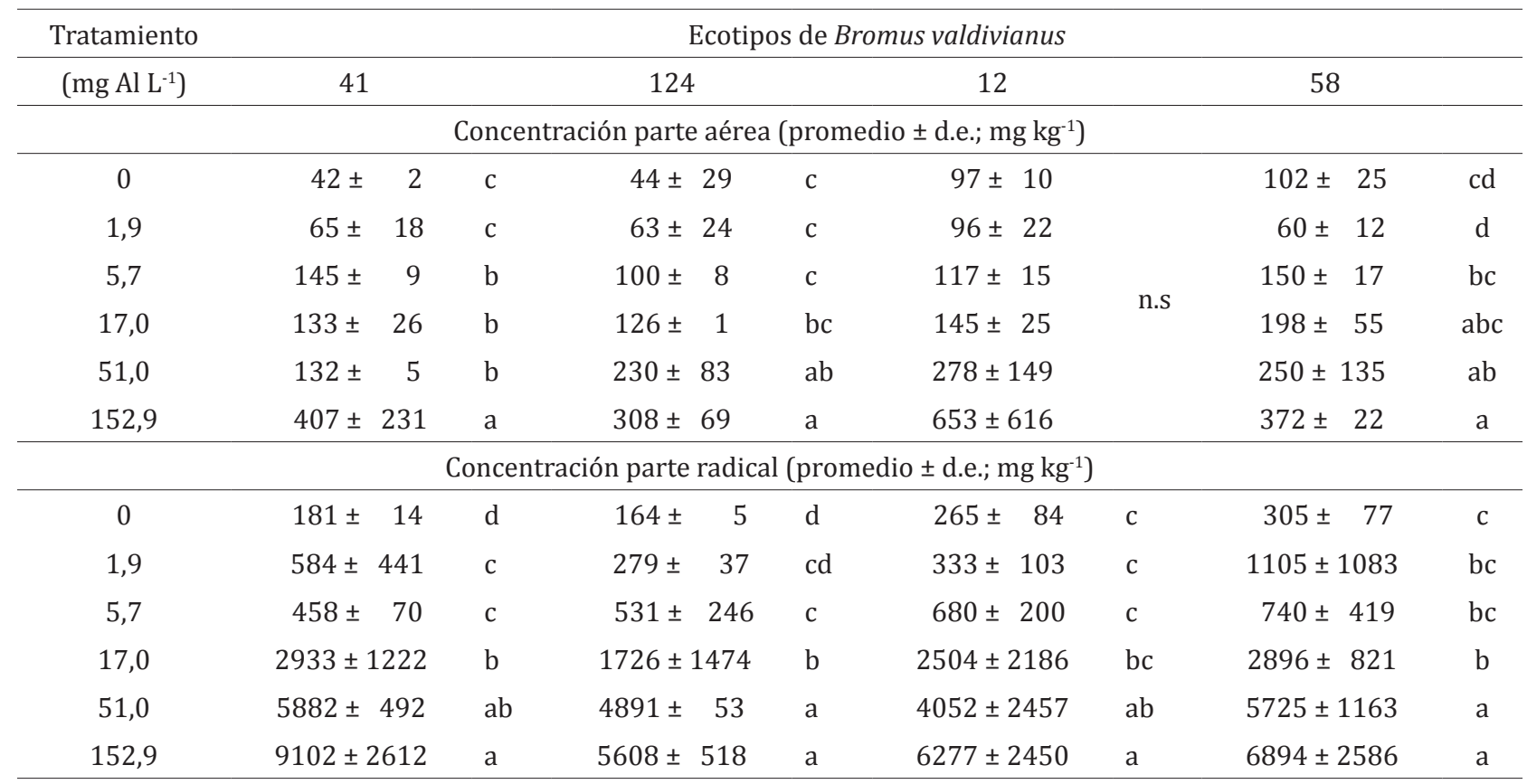

Diferentes letras dentro de cada columna indica diferencias significativas (p-valor $\leq 0,05$; Duncan). n.s. indica diferencias no significativas ( $p \geq 0,05$; Duncan); d.e.= Desviación estándar.

que tendió a aumentar en la medida que aumentó la concentración en solución, o simplemente a que este ecotipo de bromo presenta un comportamiento lineal, no incrementando su concentración en la parte aérea a pesar de que existan mayores concentraciones en la parte radical, a un mismo nivel de tratamiento.

En el cuadro 3, se observó que la concentración de aluminio radical, tiene un comportamiento similar a la concentración de aluminio de la parte aérea, donde a mayor concentración de aluminio en solución ma- 
yor concentración de aluminio en la biomasa radical. Similares resultados encontraron Rengel y Robinson (1989). Por otro lado, Akhter et al. (2009) señalan que en un experimento con sorgo en solución nutritiva, los resultados indicaron que entre las menos tolerantes al $\mathrm{Al}$, la mayor tolerancia estaba dada por una menor absorción de Al en las raíces. Marín-Garza et al. (2010), señalan que en un experimento con arroz en solución nutritiva, las concentraciones de $\mathrm{Al}$ en la parte aérea aumentaron en relación al aumento de la concentración de Al en solución. Además, existió interacción entre variedad y concentración de $\mathrm{Al}$, indicando que existen diferencias en tolerancia al $\mathrm{Al}$, de acuerdo a la variedad evaluada.

Las diferencias en concentración de aluminio para la parte radical en las gramíneas evaluadas, fueron estadísticamente significativas para cada ecotipo de bromo ante los distintos tratamientos de Al en solución. Para los ecotipos de bromo 41 y 124 se describieron cuatro rangos de concentración estadísticamente significativos en relación al aluminio aplicado a la solución, en tanto que para los ecotipos de bromo 12 y 58 se presentaron sólo tres rangos de concentraciones estadísticamente significativos.

Los ecotipos evaluados presentan un diferente rango, estadísticamente significativo, para el aumento en concentración de aluminio radical. Al relacionar esta concentración de Al radical con la producción de materia seca aérea y radical, se puede ver que existe un mismo comportamiento entre ambas MS y la concentración de Al. Donde los ecotipos más tolerantes disminuyen la MS producida en dosis posteriores, que la dosis donde aumentó la concentración de Al radical. Por otro lado, en los ecotipos más sensibles la MS disminuye en el mismo o un menor tratamiento, que para el aumento en la concentración de $\mathrm{Al}$ en la parte radical. Las diferencias en concentración de aluminio entre la parte aérea y radical, son estadísticamente significativas y corrobora lo establecido por otros investigadores (Rengel y Robinson, 1989; Marschner, 1991). De esta forma, este comportamiento parece indicar que el mecanismo de tolerancia en las raíces de las gramíneas puede ser el factor de control de la toxicidad. El mecanismo de tolerancia de los ecotipos evaluados, se mostraría con el aumento de concentración de aluminio en las raíces, limitando su paso hacia la parte aérea. Corroborando lo anterior, Osaki et al. (1997), determinó una clasificación de tolerancia al aluminio, basado sobre el criterio de acumulación. Bajo este criterio, clasificó a las plantas como: Al-excluyentes, $\mathrm{Al}$-acumuladora radical y Al-acumuladoras. Vardar y Ünal (2007) clasifica la resistencia al Al por exclusión o tolerancia. Todo lo anterior, sugeriría que los ecotipos de bromo evaluados serian clasificados como Al-acumuladores radicales.

Dentro de los ecotipos de bromo, el 41 (clasificado como tolerante) presentó el mayor grado de tolerancia al aluminio en solución. Ello se evidencia, porque luego de un incremento inicial de la concentración de $\mathrm{Al}$ en la parte aérea (hasta $5,7 \mathrm{mg} \mathrm{Al} \mathrm{L}^{-1}$ ) se produce una estabilización de la concentración, hasta un valor umbral en que vuelve a aumentar (hasta 152,9 $\mathrm{mg} \mathrm{Al} \mathrm{L}^{-1}$ ) (figura 1). Cabe recordar que este ecotipo presentó la mayor producción de MS, además, de la mayor concentración de aluminio radical, la cual aumentaba en forma cuadrática a lo largo de todos sus tratamientos, comportamiento que difiere de la parte aérea. El ecotipo 12 fue el que se presentó más sensible, debido a que no hubo limitación para la translocación de aluminio hacia la parte aérea, mostrando un comportamiento lineal a lo largo de todos los tratamientos, además, de una mayor

Cuadro 4. Coeficientes de regresión y determinación para concentración de aluminio en la parte aérea y radical.

Table 4. Regression and determination coefficients for shoot and root aluminum concentrations.

\begin{tabular}{lccccc}
\hline & \multicolumn{3}{c}{ Parámetros de la ecuación ajustada } \\
\hline Ecotipos & A & B & C & D & \\
\hline & & & Aluminio aéreo & \\
\hline Bromo 41 & 89,76 & 0,135 & $9,954 \mathrm{E}^{-16}$ & 7,949 & 0,956 \\
Bromo 124 & 69,00 & 3,934 & $-0,01549$ & - & 0,997 \\
Bromo 12 & 95,07 & 3,635 & - & - & 0,998 \\
Bromo 58 & 111,3 & 3,552 & $-0,01216$ & 0,899 \\
\hline Bromo 41 & & & Aluminio radical & - & 0,992 \\
Bromo 124 & 56,37 & 146,8 & $-0,5738$ & - \\
Bromo 12 & 50,00 & 120,5 & $-0,5499$ & - & 0,996 \\
Bromo 58 & 292,1 & 98,48 & $-0,3889$ & - & 0,981 \\
\hline
\end{tabular}




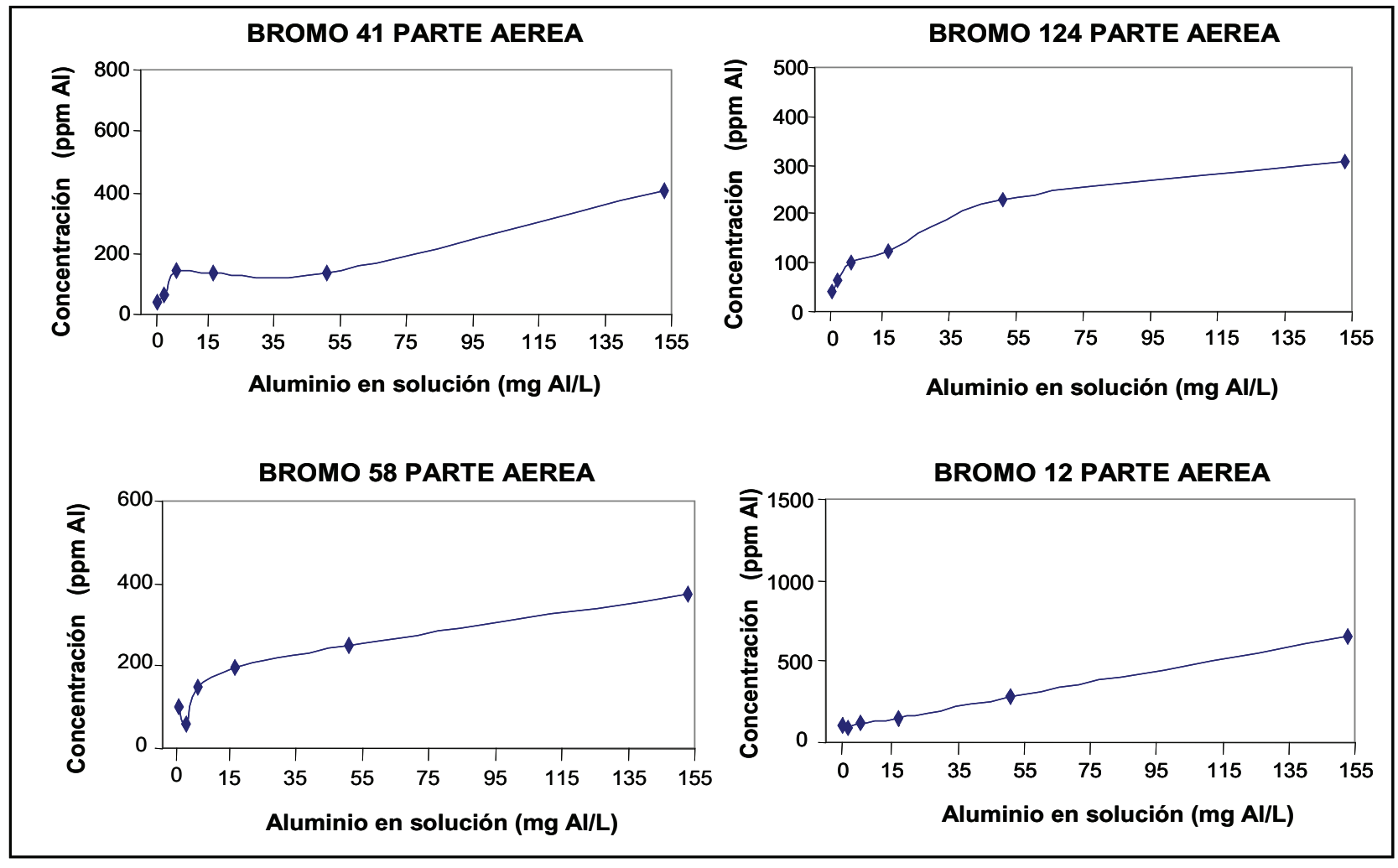

Figura 1. Concentración de aluminio en la parte aérea a través de los distintos tratamientos, para los ecotipos de bromo 41 , 124,58 y 12 .

Figure 1. Aluminium concentration in plant shoots subjected to different treatments, for ecotypes 41, 124, 58 and 12 of Bromus valdivianus.

concentración de Al. Los ecotipos de bromo 124 y 58 mostraron un comportamiento cuadrático en la parte aérea, de la misma forma que en la parte radical, diferenciándose en la producción de MS obtenida y la concentración de Al acumulada. Diferencias en tolerancia al aluminio para diferentes cultivares de una misma especie de gramíneas, fueron encontradas por Wheeler et al. (1992a) y Wheeler et al. (1992b). Además, Garvin y Carver (2003); George et al. (2012) y Ma et al. (2014) explican las diferencias en tolerancia al $\mathrm{Al}$ entre especies.

En relación a las ecuaciones ajustadas para producción de materia seca y concentración de aluminio, se utilizaron promedios de los datos debido a que las ecuaciones fueron una herramienta utilizada para describir el comportamiento de las gramíneas a través de los distintos tratamientos de aluminio en solución y no son utilizadas como una herramienta de predicción.

Por todo lo anterior, se sugiere que en estudios posteriores se utilicen rangos de aluminio en solución, entre $1,9 \mathrm{mg} \mathrm{Al} \mathrm{L}^{-1}$ y $17,0 \mathrm{mg} \mathrm{Al} \mathrm{L}^{-1}$. Para poder determinar con mayor precisión los umbrales de pérdida en producción de materia seca y de aumento en la concentración de aluminio en las plantas.

\section{CONCLUSIÓN}

La concentración crítica de aluminio en solución para los ecotipos de bromo evaluados está entre 5,7 y $17 \mathrm{mg} \mathrm{Al} \mathrm{L}^{-1}$. En este rango se produciría una pérdida significativa en producción de materia seca aérea para todos los ecotipos de Bromus valdivianus evaluados. En el primer tramo $0-1,9 \mathrm{mg} \mathrm{Al} \mathrm{L}^{-1}$, existe una aparente pérdida de producción de MS aérea que es más acentuada en los tolerantes que los menos tolerantes y sensibles, por el contrario, en los sensibles hay incremento de la producción, pero que no es significativa. Mientras que el rango crítico para la concentración de aluminio radical en todos los ecotipos de $B$. valdivianus, dependería del grado de tolerancia del ecotipo evaluado. Sugiriendo que previo a la pérdida en productividad de materia seca existiría un aumento en la acumulación de aluminio radical para los ecotipos tolerantes, no presentando este comportamiento los ecotipos sensibles.

Todas las gramíneas evaluadas presentarían un mecanismo de tolerancia Aluminio-acumuladora radical, sugerido por la mayor acumulación del aluminio en la parte radical que en la parte aérea. Dentro de este tipo 
de tolerancia, los ecotipos de B. valdivianus varían en su estrategia. Los ecotipos más tolerantes tienden en mayor medida, a evitar el traspaso de aluminio desde las raíces hacia la parte aérea, en tanto que los más sensibles no presentarían esta restricción.

\section{AGRADECIMIENTOS}

Se agradece a los pares revisores por los comentarios y sugerencias que contribuyeron positivamente a esta publicación.

\section{REFERENCIAS}

Akhter, A., Khan, M.S.H., Hiroaki, E., 2009. Greater contribution of low-nutrient tolerance to sorghum and maize growth under combined stress conditions with high aluminum and low nutrients in solution culture simulating the nutrient status of tropical acid soils. Soil Science and Plant Nutrition 55(3), 394-406.

Barceló, J., Poschenrieder, CH., Vázquez, M.D., Gunsé, B., 1996. Aluminium phytotoxicity. A challenge for plant scientists. Fertilizer research 43, 217-223.

Bennet, R.J., Brenn, C.M., 1991. The aluminium signal: New dimensions to mechanisms of aluminium tolerance. Plant and Soil 134,153-166.

Blanco, J., Balocchi, O., López, I., 2010. Variabilidad fenotípica en accesiones Bromus valdivianus Phil. de la provincia de Valdivia. Agro Sur 38(2), 68-79. doi:10.4206/agrosur.2010.v38n2-02

Brady, N., Weil, R., 2008. The nature and properties of soils. Pearson Prentice Hall, Upper Saaddle River, New Jersey.

Broadly, M., Brown, P., Cakmak, I., Feng Ma, J., Rengel, Z., Zhao, F., 2012. Adaptation of plants to adverse chemical soil conditions, in: Marschner, P. (Ed.), Mineral Nutrition of Higher Plants. $3^{\text {rd }}$ Edition. Academic Press, Sydney, pp. 249-270.

De Macedo, F.L., Pedra, W.N., Da Silva, S.A., 2011. Effect of aluminum in plants of Jatropha curcas L. grown in nutritive solution. Semina-Ciencias Agrarias 32(1), 157-163.

Forde, M.B., Edgar, E., 1995. Checklist of pooid grasses naturalised in New Zealand. Tribes Bromeae and Brachypodieae. New Zealand Journal of Botany 33, 35-42.

Foy, C.D., 1984. Physiological effects of hydrogen, aluminium and manganese toxicities in acid soil, in: Pearson and Adams (Eds.), Soil acidity and liming. $2^{\text {nd }}$ Edn. American Society of Agronomy, Wisconsin, pp. 57-97.

Garvin, D., Carver, B., 2003. Role of the genotype in tolerance to acidity and aluminium toxicity, in: Rengel, Z. (Ed.), Handbook of soil acidity. Marcel Dekker, New York, pp. 387-406.

George, E., Horst, W., Neumann, E., 2012. Adaptation of plants to adverse chemical soil conditions, in: Marschner, P. (Ed.), Mineral Nutrition of Higher Plants. $3^{\text {rd }}$ Edition. Academic Press, Sydney, pp. 409-472.

Hayes, R.C., Scott, B.J., Dear, B.S., 2011. Seedling validation of acid soil tolerance of lucerne populations selected in solution culture high in aluminium. Crop \& Pasture Science 62(9), 803-811.

Havlin, J.L., Beaton, I.D., Tisdale, S.L., Nelson, W.L., 2005. Soil
Fertility and Fertilizers, $7^{\text {th }}$ Edition. Prentice Hall, New Jersey.

Kinraide, T.B., 1993. Aluminium enhancement of plant growthin acid rooting media. A case of reciprocal alleviation of toxicity by two toxic cations. Physiologia Plantarum 88, 619-625.

Kochian, L., 1995. Cellular mechanisms of aluminium toxicity and resistance in plants. Annual Review of Plant Physiology 46, 237-260.

Kochian, L., Piñeros, M.A., Hoekenga, O.A., 2005. The physiology, genetics and molecular biology of plant aluminum resistance and toxicity. Plant and Soil 274, 175-195.

Lee, J., Pritchard, M.W., 1984. Aluminium toxicity expression on nutrient uptake, growth and root morphology of Trifolium repens L. cv. "Grassland Huia”. Plant and Soil 82, 101-116.

López, I., Balocchi, O., Lailhacar, P., Oyarzun, C., 1997. Caracterización de sitios de crecimiento de seis especies pratenses nativas y naturalizadas del Dominio Húmedo de Chile. Agro Sur 25(2), 62-80.

Ma, J.F., Chen, Z.C., Shen, R.F., 2014. Molecular mechanisms of Al tolerance in gramineous plants. Plant and Soil 381, 1-12.

Marín-Garza, T., Gómez-Merino, F., Trejo-Téllez, L., MuñozOrozco, A., Tavitas-Fuentes, L., Hernández-Aragón, L., Santacruz-Varela, A., 2010. Respuestas fisiológicas y nutrimentales de variedades de arroz a la concentración de aluminio. Revista Fitotécnia Mexicana 33(1), 37-44.

Marschner, H., 1991. Mechanisms of adaptation of plants to acid soils. Plant and Soil 134, 1-20.

Marschner, H., 1995. Mineral Nutrition of Higher Plants. $2^{\text {th }}$ ed. Academic Press. San Diego, USA.

Matthei, O., 1986. El género Bromus L. (Poaceae) en Chile. Gayana Botánica 43(1-4), 47-110.

Menzies, N., 2003. Toxic elements in acid soils: Chemistry and Measurement. In: Handbook of soil acidity. Rengel, Z. (Ed.), Marcel Dekker. New York, USA, pp. 267-296.

Mossor-Pietraszewska, T., 2001. Effect of aluminium on plant growth and metabolism. Acta Biochemica Polonica 48(3), 673-686.

Osaki, M., Watanabe, T., Tadano, T., 1997. Benefical effect of aluminum on growth of plants adapted to low $\mathrm{pH}$ soils. Soil Science Plant Nutrition 43(3), 551-563.

Rengel, Z., Robinson, D.L., 1989. Aluminium effects on growth and macronutrient uptake by annual ryegrass. Agronomy Journal 81, 208-215.

Silva, S., 2012. Aluminium Toxicity Targets in Plants. Journal of Botany 2012, 1-8.

Stewart, A., 1996. Potential value of some Bromus species of the section Ceratochloa. New Zealand Journal of Agricultural Research 39, 611-618.

Toneatti, M., 2006. Evaluación de la tolerancia a aluminio en 125 accesiones de bromo (Bromus valdivianus Phil.). Tesis de Magíster en Ciencias Mención Producción Animal, Universidad Austral de Chile. 123 p.

Vardar, F., Ünal, M., 2007. Aluminum toxicity and resistance in higher plants. Advances in Molecular Biology 1, 1-12.

Wheeler, O.M., Edmeades, D.C., Christie, R.A., Gardner, R. 1992a. Effects aluminium on growth of 34 plant species: a summary of results obtained in low ionic strength solution culture. Plant and Soil 146, 61-66. 
Wheeler, O.M., Edmeades, D.C., Smith, D.R., Wedderburn, M.E. $\quad$ Yang, Z.B., Rao, I.M., Horst, W.J. 2013. Interaction of alumi1992b. Screening perennial rye-grass from New Zealand for aluminium tolerance. Plant and Soil 146, 9-19. nium and drought stress on root growth and crop yield on acid soils. Plant and Soil 372, 3-25. 
